\title{
Écosystème
}

\section{UNE MUSIQUE AFFECTANTE, TENTATIVE DE PRISE DE CONTACT}

\section{Raphaël Brunel}

Volume 1, numéro 1, 2018

Musique

URI : https://id.erudit.org/iderudit/1058618ar

DOI : https://doi.org/10.7202/1058618ar

Aller au sommaire du numéro

Éditeur(s)

La chambre blanche

ISSN

2562-3222 (numérique)

Découvrir la revue

Citer cet article

Brunel, R. (2018). UNE MUSIQUE AFFECTANTE, TENTATIVE DE PRISE DE

CONTACT. Écosystème, 1(1), 21-26. https://doi.org/10.7202/1058618ar d'utilisation que vous pouvez consulter en ligne.

https://apropos.erudit.org/fr/usagers/politique-dutilisation/ 


\section{UNE MUSIQUE AFFECTANTE, TENTATIVE DE PRISE DE CONTACT}

\section{Raphaël Brunel}

Régulièrement, la découverte d'exoplanètes localisées dans la zone dite d'habitabilité de leur étoile, où l'eau est susceptible d'exister à l'état liquide, ravive l'hypothèse du développement d'une forme de vie extraterrestre ainsi que cette question restée jusqu'à présent sans réponse : sommes-nous seuls (nous, humains et non-humains) dans l'univers ? L'équipe de chercheurs qui a révélé au début de l'année 2017 la présence de trois de ces planètes en orbite autour de Trappist-1, une "naine ultra-froide » située à 39 annéeslumière de la Terre dans la constellation du Verseau ${ }^{32}$, estime qu'elle sera en mesure de découvrir d'ici dix ans si celles-ci sont ou non « habitées ».

Si une existence extraterrestre était un jour avérée et se révélait "intelligente », nous pourrions aisément imaginer qu'une fois digérées les implications scientifiques philosophiques et morales d'une telle nouvelle, une fois les premières observations entreprises, l'étape suivante (peut-être insurmontable) dans notre appréhension de cette nouvelle et a priori radicale altérité consisterait à amorcer une prise de contact. Une tentative de communiquer, d'engager un échange, quelle qu'en soit la nature, semble poser, comme prérequis la question du langage et de la traduction, des interfaces et médiations nécessaires à un décryptage. Mais il n'est pas exclu que cette entité n'ait pas développé de moyens d'expression ou qu'on ne soit pas en mesure de les identifier ou de les décoder.

Certains scénarios de films de science-fiction se sont attachés à mettre en scène cette rencontre, généralement amicale et pacifiste, et à en imaginer les premiers échanges. Quand l'extraterrestre, grâce à un don extrasensoriel ou à l'avancée de sa technologie, ne s'exprime pas déjà dans un anglais parfaitement maîtrisé, son langage prend du moins selon les cas une forme orale, écrite, gestuelle, voire tactile ou psychique. Ainsi pour prendre un exemple récent, dans Arrival (2016) de Denis Villeneuve, une linguiste de renom, pour qui le langage à titre de fondement de toute civilisation est « la première arme engagée dans un conflit», tente d'établir le lien avec des «heptapodes» dont les vaisseaux sont soudainement apparus en plusieurs points du globe. Si les sonorités qu'ils émettent ne permettent pas d'envisager une communication verbale, le personnage interprété par Amy Adams va progressivement réussir à percer le mystère des inscriptions circulaires complexes qui caractérisent leur écriture et découvrir la raison de leur venue sur Terre, c'est-à-dire transmettre aux humains leur langue, dont le rapport non linéaire au temps permet de voir le futur. Par un phénomène de révélation quasi mystique, la chercheuse devient dépositaire d'une langue qu'elle qualifie, dans l'ouvrage qu'elle lui consacre et qu'on aperçoit à la fin du film, d' « universelle ».

Cette question d'un langage commun permettant de communiquer avec des êtres venus du fond de la galaxie était déjà au coeur de l'inoubliable Close Encounter of the Third Kind réalisé en 1977 par Steven Spielberg, qui apparaît comme le parangon du genre. Mais le dialogue qui s'établit entre humains et extraterrestres lors de la monumentale scène finale

32 Michaël Guillon et al., « Seven Temperate Terrestrial Planets around the Nearby Ultracool Dwarf Star TRAPPIST$1 »$, Nature, no. 542, février 2017, p. $456-460$. 
qui voit l'arrivée d'une navette spatiale au-dessus de la Devils Tower, ne repose pas ici sur une forme d'écriture, d'oralité ou d'extrasensorialité, mais sur un code musical de cinq notes imaginé par le compositeur John Williams avant même le tournage du film. Ce signal (Ré Mi Do Do Sol) constitue le phrasé de base à partir duquel va se mettre en place un échange musical, un jeu de question-réponse de plus en plus intense et virtuose, les hommes reproduisant à l'aide d'un synthétiseur ARP 2500 les orchestrations graves (tuba/contrebasson) émises par le vaisseau, dont les habitants donnent ainsi, comme le suggère l'un des scientifiques présents sur place, leur première leçon de « vocabulaire tonal ».

Avec Spielberg, la musique constitue donc l'interface (par le biais de la mélodie, mais aussi de l'évolution technologique instrumentale que représente le synthétiseur ${ }^{33}$ ) permettant d'établir un contact inter espèce pacifiste et bien au-delà, un langage universel, un mode de communication médian, à partir duquel il semble possible de se comprendre.

Hors de cette communion (science) fictionnelle, la musique peut-elle vraiment être envisagée comme un langage universel ? À l'échelle de l'espèce humaine, on en trouve la trace, comme l'ont montré l'ethnomusicologie et l'archéologie, aux quatre coins de la planète et dans des civilisations très anciennes. Le plus vieil instrument découvert - une flûte taillée dans un os animal — date de 35000 ans. Le paléontologue anglais Steven Mithen va jusqu'à émettre le postulat que la musique, sous une forme bien différente de celle que nous connaissons aujourd'hui, composée d'un ensemble de chants, de bruits et de rythmes produits par le corps, aurait précédé l'apparition du langage verbal en tant que mode d'expression individuelle et collective et aurait favorisé son développement ${ }^{34}$. Au cours de l'histoire de l'évolution, les capacités musicales auraient donc, selon lui, été inscrites dans le génome humain et relèveraient d'un processus à la fois culturel et biologique. Si les neurosciences ont démontré que la musique et le langage activent des zones du cerveau communes, ils n'empruntent toutefois pas les mêmes logiques en matière de transmission. Au risque de caricaturer un peu les choses, là où le langage constitue un système de communication véhiculant une donnée et un sens, la musique semble davantage porteuse d'affects. Au sens « objectif» et verbalisable de l'information, elle privilégierait donc l'effet, qui pourrait cependant varier en fonction de l'auditeur, de son origine, des conventions musicales et normes interprétatives.

Ainsi, si une forme de vie extraterrestre est un tant soit peu sujette à des mécanismes similaires, ce qui reste peu probable, il faudrait donc imaginer l'interface sonore moins comme le sésame d'une signification ou d'une grammaire secrète que comme une adresse subjective et affective, ou plutôt affectante, et on abandonnerait alors la passion candide de Spielberg pour l'ufologie, pour l'humour noir du Mars Attacks! de Tim Burton (1996) où

33 L'invention et le recours à certains instruments (synthétiseur, sampler, platine vinyle, etc.) a participé à élargir les perspectives de la musique et permis l'expérimentation de sons nouveaux, dans un rapport fusionnel entre l'homme et sa machine, tout en développant un imaginaire singulier en rupture avec toute effet de réalité, notamment dans les musiques africaines-américaines associées à l'afrofuturisme. À ce sujet, voir Kodwo Eshun, More Brilliant than the Sun : Adventures in Sonic Fiction, Londres, Quartet Books, 1998.

34 Voir Steven Mithen, The Singing Neanderthals. The Origins of Music, Language, Mind and Body, Cambridge, Harvard University Press, 2006. 
une chanson du répertoire country, Indian Love Call (1952) de Slim Whitman, devient la solution miracle pour se débarrasser des martiens ricanants qui ont décidé d'envahir la Terre.

Évidemment, tout ceci reste spéculatif et relève du domaine de la fiction. Il ne s'agit pas tant de prouver la potentielle universalité de la musique, que d'envisager en quoi le sonore, justement parce qu'il repose sur une forme de transmission qui dépasse le verbe et génère des effets d'ordre physique, psychologique ou biologique, pourrait jouer le rôle d'interface privilégiée, de vecteur possible ou fantasmé dans une communication entre l'homme et son autre. Dans cette perspective, l'hypothétique extraterrestre du cinéma pourrait laisser place à une forme de vie bien terrestre qui est loin d'avoir livré tous ses mystères bien qu'elle représente près de $90 \%$ de la biomasse ${ }^{35}$ et garantit les conditions d'existence des différentes espèces qui y évoluent.

Pour le botaniste Francis Hallé, les plantes constituent une altérité fondamentale par rapport à l'homme et plus généralement à l'animal : « Je ne suis pas zoologiste, mais présentez-moi n'importe quel animal et je parviendrai à savoir ce qu'il mange, s'il voit, comment il se reproduit. Il y aura forcément une interaction : je peux lui faire peur, le faire fuir... alors qu'une plante n'a ni queue ni tête. Elle ne fait aucun bruit. Comment s'exprime-t-elle ? » ${ }^{36}$ Paru en 1973 et adapté en film documentaire en 1979, le livre The Secret Life of Plants de Peter Tompkins et Christopher Bird tente en partie de percer l'énigme de cette intériorité végétale $^{37}$. Les deux auteurs reviennent notamment sur l'effet Backster. En 1966, Cleve Backster, un ancien membre de la CIA spécialisé dans le détecteur de mensonges, branche son polygraphe sur les feuilles d'une plante verte et constate que celle-ci réagit lorsqu'il l'arrose. Poussant plus loin l'expérience, il décide de brûler à l'aide d'une allumette une des feuilles, mais l'aiguille de l'appareil s'emballe avant qu'il n'exécute son geste, comme si la plante avait anticipé son intention. Il en déduit que les végétaux sont doués d'affects et de conscience, qu'ils sont sensibles aux émotions et actions humaines. Ces conclusions restent cependant controversées et n'ont jamais été validées par le monde scientifique. Francis Hallé, quant à lui, met en garde contre le risque d'un anthropomorphisme qui consisterait à appliquer au monde végétal des notions humaines comme celles de conscience, de sensibilité ou d'intelligence, ce qui risquerait par des effets de projection de nous aveugler sur ses spécificités. Il évoque toutefois une plante découverte dans un jardin botanique chinois, la codariocalyx motorius (ou desmodium gyrans), surnommée en anglais telegraph plant ou semaphore plant, qui a la particularité de s'animer, de « danser » sous l'effet d'un bruit ou d'une musique, les folioles latérales de chaque feuille se mettant à bouger ${ }^{38}$. Si un tel phénomène semble induire qu'elle est dotée de capteurs sonores, les mécanismes précis de cette mise en mouvement restent inexpliqués.

\footnotetext{
35 Francis Hallé, Éloge de la plante : Pour une nouvelle biologie, Paris, Seuil, 2014, p. 321. devenu botaniste », dans http://www.liberation.fr/debats/2016/12/29/francis-halle-une-plante-n-a-ni-queue-ni-tete-cest-pour-cette-alterite-que-je-suis-devenu-botaniste 1538044 Libération, 29 décembre 2016. Page consultée le $25 / 01 / 18$

37 Peter Tompkins et Christopher Bird, The Secret Life of Plants, New York, Harper \& Row, 1973.

38 Francis Hallé, Atlas de botanique poétique, Paris, Arthaud, 2016.
} 
En soulevant l'hypothèse d'une interaction possible entre les actions et sentiments humains et le développement et le bien-être des plantes, la parution de The Secret Life of Plants semble avoir suscité des vocations chez certains musiciens. Si les végétaux avaient déjà pu être incorporés comme instruments dans certaines compositions contemporaines, telles que Child of Tree (1975) de John Cage, un solo pour percussion recourant à l'amplification de feuilles, de branches ou de cactus ${ }^{39}$, ils deviennent alors l'objet d'une adresse spécifique, les premiers destinataires d'une musique conçue pour eux. Au milieu des années 1970 se multiplient ainsi les disques, aux tonalités volontiers easy-listening, censées agir sur la croissance et le bien-être des plantes: Music to Grow Plants de Dr George Milstein (1970), Mother Earth's Plantasia de Mort Garson (1976), Plant Music de Baroque Bouquet (1976), Plant Talk de Holly Roth (1976) ou encore Rhapsody in Green de Roger Roger, sur la pochette duquel on peut lire: "L'éditeur se fait un plaisir de vous offrir un disque spécialement conçu pour vos plantes. Cette musique écrite par Roger Roger, est un des sésames qui vous ouvrira les chemins d'une communication avec les végétaux. »

En 1992, le physicien et chercheur français Joël Sternheimer (qui a eu une carrière de chanteur dans les années 1960 sous le pseudonyme d'Evariste) dépose le brevet du procédé de régulation épigénétique de la synthèse protéique, censé notamment expliquer l'influence de la musique sur des organismes vivants et sur la croissance des plantes en particulier. Selon lui, les acides aminés émettent, au moment où ils s'assemblent pour former des protéines, des ondes de nature quantique, les ondes d'échelle, dont les fréquences peuvent être transposées en notes de musique. Une protéine peut donc être associée à une mélodie qui lui est propre - ce qu'il appelle "protéodie» — et qui, si elle était jouée régulièrement, stimulerait —ou inhiberait — son développement. Là encore, ces conclusions semblent diviser la communauté scientifique, mais posent aussi en creux, de part et d'autre, la question de la traduction d'un signal, par le biais d'un système musical voire d'une interface technologique chez l'homme et d'un processus biologique chez la plante.

Hésitant entre le rationnel et l'irrationnel, à cheval entre la botanique, les parasciences et l'esprit New Age, les liens qui tentent de se tisser entre la musique et le végétal ne pouvaient manquer de susciter l'intérêt d'artistes contemporains. Ainsi en 2007 au Palais de Tokyo, Peter Coffin rejoue avec le projet Music for Plants certaines des expériences musicales des années 1970, en installant Untitled (Greenhouse) (2002), une serre regroupant une diversité de plantes et envisagée comme lieu de culture, dans les différents sens du terme. Guitare, ampli et micro y étaient mis à disposition du public afin d'improviser quelques notes à destination de cette flore hétérogène et certains musiciens et artistes, tels que Vincent Epplay, Davide Balula ou Pierre-Yves Macé, étaient régulièrement conviés à établir un dialogue avec cette matière vivante et agir sur sa croissance, une expérience qui donna également lieu à une émission radiophonique avec Clocktower à New York ${ }^{40}$. A l'occasion de l'exposition, Mais qu'est-il arrivé à cette musique? à la Villa Arson en 2008, Arnaud Maguet proposait quant à lui avec Mind

39 Cette oeuvre est initialement composée pour accompagner une chorégraphie de Merce Cunningham intitulée Solo. En 1976, Cage conçoit dans une veine similaire Branches, cette fois pour plusieurs interprètes dont le nombre peut varier selon les présentations.

40 «Peter Coffin : Music for Plants », dans http://clocktower.org/show/peter-coffin-music-for-plants. Page consultée le 25/01/18. 
Garden une version plus licencieuse de ces procédés de culture par la musique, en créant un jardin de plantes psychoactives bercées par une bande-son signée Bader Motor (Fred Bigot, Vincent Epplay, Arnaud Maguet) dont le titre, Musique pour les plantes des dieux, fait directement écho à l'ouvrage Plants of the Gods de Richard Evan Schultes et Albert Hofmann (le père du LCD), retraçant l'usage et les significations symboliques ou spirituelles de tels végétaux à travers les âges et les civilisations. L'oeuvre évoque ainsi un double effet, qui ne constitue peut-être qu'un seul et même mouvement, rétroactif : celui de la musique sur les plantes, et de certaines plantes sur l'esprit humain et sa production musicale.

Avec leur film Conversation avec un cactus (2017), Elise Florenty et Marcel Türkowsky suivent une jeune femme qui cherche à réactiver dans le Tokyo contemporain les expériences menées dans les années 1970 par le couple Hashimoto ${ }^{41}$ visant à communiquer avec un cactus par l'intermédiaire, comme Backster, d'un détecteur de mensonges. Entre fiction et documentaire, ils reviennent sur la réception, notamment télévisée, de cette aventure, prise entre fascination et détournement comique, et installent le climat d'une enquête policière, le personnage principal cherchant à apprendre à parler au cactus et à révéler son langage intime dans la perspective qu'il puisse servir à l'avenir de potentiel témoin lors d'un crime. Si certaines scènes font écho à la vidéo Teaching a Plant the Alphabet (1972) de John Baldessari, Florenty et Türkowsky privilégient à l'ironie de l'action absurde de l'artiste californien, une narration onirique qui met en tension certaines contradictions de la société japonaise, où les traditions animistes appellent à multiplier les communications entre humains et non-humains, mais où la pression des rapports sociaux ne permet pas toujours de s'exprimer en pleine conscience.

Au-delà du pouvoir de fascination qu'exerce la possibilité de communiquer avec des plantes, cette altérité radicale, l'intérêt des artistes pour cette histoire parallèle de la communication s'attache probablement moins à l'impact de la création humaine sur son milieu qu'à l'occasion qu'elle offre de redéfinir les jeux d'influences entre l'homme et son environnement, en brouillant la séparation nette entre ce qui relève de la nature et de la culture. Pour le philosophe Emanuele Coccia, la plante est « le lieu d'indifférence entre le biologique et le culturel, le logos et l'extension », elle est " une cosmogonie en acte, la genèse constante de notre $\operatorname{cosmos}^{42} »$, car elle produit l'atmosphère dont les êtres terrestres ont besoin pour vivre, elle constitue notre univers. Et la musique, peut-être, nous aidera-telle à appréhender notre univers, à affecter et avant tout à être affecté, à rester sensible aux réseaux de relations dans lesquels nous ne cessons d'être pris.

\footnotetext{
41 Directeur de Fuji Electronics, M. Hashimoto est à l'origine de plus de 200 inventions dont les panneaux lumineux LED.

42 Emanuele Coccia, La Vie des plantes, une métaphysique du mélange, Paris, Payot \& Rivages, 2016, p. 22.
} 


\section{Références}

COCCIA, Emanuele. La Vie des plantes, une métaphysique du mélange. Paris, Payot \& Rivages, 2016. $191 \mathrm{p}$.

HALLÉ, Francis. Atlas de botanique poétique. Paris, Arthaud, 2016. 144 p.

HALLÉ, Francis. Éloge de la plante : Pour une nouvelle biologie. Paris, Seuil, 2014. 354 p.

MITHEN, Steven. The Singing Neanderthals : The Origins of Music, Language, Mind and Body. Cambridge, Harvard University Press, 2006. 384 p.

TOMPKINS, Peter et Christopher Bird. The Secret Life of Plants. New York, Harper \& Row, 1973. $402 \mathrm{p}$. 